\title{
THE PROTOTYPE OF A TRAFFIC SIGNS RECOGNITION SYSTEM USING MOBILE AUGMENTED REALITY
}

\author{
Florentin - Alexandru DIȚĂ \\ Bucharest University of Economic Studies, Romania \\ alexandru.dita@csie.ase.ro \\ Mihaela - Irina ENĂCHESCU \\ Bucharest University of Economic Studies, Romania \\ irina.enachescu@csie.ase.ro
}

\begin{abstract}
In this paper is proposed a traffic signs recognition prototype based on template matching approach of the 3D Registration technique in order to make use of the benefits provided by mobile augmented reality. The proposed solution finds speed traffic signs, extracted from a mobile device camera used in real environment, and uses them as AR markers to augment the virtual elements. This information is meant to help the driver during driving process by giving him various audio or visual indications via his mobile device. As one of the top three causes of road accidents is the distracted driving, usually caused by the driver talking on the mobile phone or sending a text message, including the audio component that gives signals when the road signs are not observed is useful. We proposed an ontology to attach some knowledge to each traffic sign, to guide its interpretation. Nevertheless, the performance of the prototype is affected by several factors, such as: video source, lighting conditions, occlusion, chaotic background and viewing angle, deterioration and deformations of the traffic signs etc. In order to reduce the negative effects of above factors is necessary to use the following methods: image resizing, grayscale transformation, median blur, Hough transformation. In the first part of the research we focused on describing the architecture of the prototype and the components of the OpenCV library, which are integrated in the current solution. In the second phase, we highlighted the most common issues that affect the performance of the application.
\end{abstract}

Keywords: augmented reality, traffic sign, OpenCV library, ontology, mobile applications JEL classification: D83, O18

DOI: $10.12948 / \mathrm{ie} 2019.06 .08$

\section{Introduction}

In the last years, the automobile driving has become an important part of daily life and the use of them as primary mode of transportation has been increasing. According to [1], the road safety has become a key challenge for industry and society due to the fact that the global volume of traffic has increased around one billion vehicles. Also, the authors highlight that "more than $80 \%$ of vehicle accidents are caused by driver error". Consequently, both large automotive companies and researchers have focused on the use of different technologies for the development of advanced driving assistant systems.

Lately, the mobile Augmented Reality (AR) is one of the most used technology in the automotive industry due to the recent performances, the benefits it offers and, also, the computing power of today's mobile devices. According to [1], [2] and [3] AR refers to the extension of the real environment with $2 \mathrm{D}$ or $3 \mathrm{D}$ virtual objects in order to create a mixed reality in real time. Moreover, [2], [3] and [4] highlight that mobile augmented reality uses 
image recognition systems and mobile device's instruments (GPS, accelerometer and gyroscope) to determine the location, orientation and direction of the device in physical environment in order to display the virtual content.

More studies, such as [5] and [6] present a traffic signs recognition system based on feature extraction. In [5] is proposed a traffic signs detection and recognition system based on colour and shape features. According to the authors the system consists in image segmentation based on HSV colour space, afterwards remove complex background interference and apply an affine transformation correction based on geometry features. Finally, using Gabor filter extract the image characteristics and identify the corresponding model. On the other hand, [6] suggests a traffic signs recognition system based on the Byte-MCT method for detection and Convolutional Neural Network (CNN) for recognition. The authors highlight that Byte-MCT method presents good performance for region detection regardless of illumination changes.

Other studies, such as [1], [7] and [8] present a traffic signs assistant system based on mobile augmented reality. In [1] is proposed a novel approach to localizing and tracking vehicles and uses 3D AR-HUD for extending the traffic signs with additional information. The authors of [7] propose a traffic signs recognition system based on the Haar cascade to reduce the computational region generation, the deep CNN approach for verification and augmented reality in order to supplements the external view with virtual information for the driver. The author of [8] highlights the benefits of an augmented reality solution for cycling based on 3D Registration.

In this paper is proposed a traffic signs recognition prototype based on template matching approach of the 3D Registration technique in order to make use of the benefits provided by mobile augmented reality. The author of [4] highlights that the template matching method extracts the global information from the frame of the real element in order to identify the target. Moreover, this method identifies both the texture less components and multiple edges targets. Also, the template matching approach presents acceptable results when the camera moves fast. The proposed solution finds speed traffic signs, extracted from the mobile device camera used in real environment, and uses them as AR markers to augment the virtual elements. This information is meant to help the driver during driving process by giving him various audio or visual indications via his mobile device. Moreover, [9] indicates that one of the top three causes of road accidents is distracted driving, usually caused by the driver talking on the mobile phone or sending a text message. Consequently, including the audio component that gives signals when the road signs are not observed proves to be useful.

In the first stage of the research we focused on describing the architecture of the prototype and the components of the OpenCV library, which are integrated in current solution. In the second phase, we aimed to highlight the major issues that affect the performance of the application.

The paper is structured as follows. The architecture of the proposed prototype is shown in Section 2. In Section 3 we describe some researches that use ontologies in driver assistance systems and we propose a specific ontology that will be queried for traffic signs interpretation. Section 4 analyses general factors that influence the performance of the system. The paper ends with conclusions and future work.

\section{Architecture}

This paper aims to introduce the architecture of a solution for traffic signs recognition, based on the template matching approach, used in augmented reality. The proposed system targets to identify traffic signs in real environment and to use the detected objects for projecting virtual information on the mobile device screen.

Before describing the solution, it is useful to understand first the use cases of the mobile application: 
- The mobile device is positioned on a fixed support attached on the car's board or to the bicycle. Using the device camera we are capturing streams containing the searched objects (traffic signs).

- The mobile application is responsible to extract the relevant information from the stream (ignoring the background and any other objects that do not represent a traffic sign), based on a complex system that uses OpenCV and other in-house built components.

- The results after the processing are encapsulated in virtual elements that in turn are augmented over the mobile device screen.

The architecture of the proposed system is shown in Figure 1.

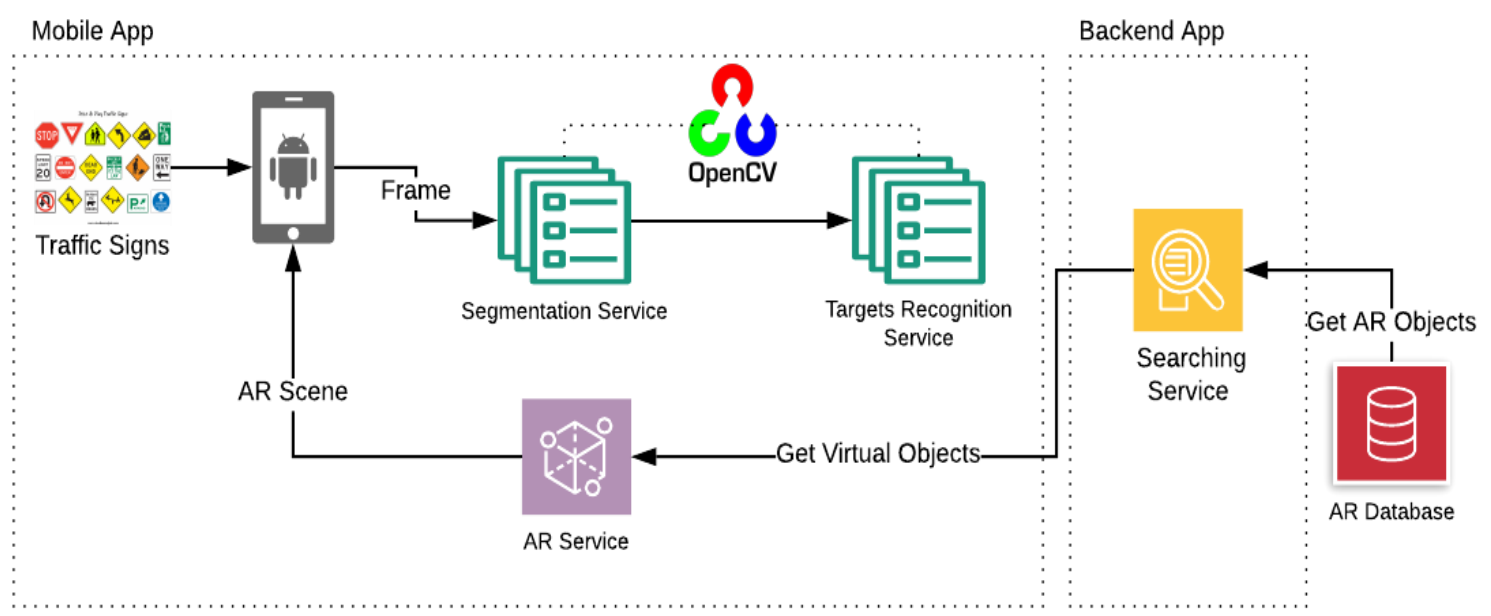

Figure 9. Architecture of the prototype

The images, captured with the device camera, represent the input data of the system. These can contain one or more traffic signs, from various categories. Each extracted frame is passed through the target objects identification process, based on the usage of the next two services: Segmentation Service and Targets Recognition Service. First of the services is responsible to establish where in the received frame is the traffic sign positioned - this is called the region of interest (ROI). Analyzing the traffic signs we notice the next particularities: they can have one of the next shapes: circle, rectangle and triangle; predominant colors are red, blue and yellow. Based on this criteria, identifying the regions of interest sums up to finding the specified shapes in each frame, and in order to achieve this the following transformations will be made on the original image: resizing to a smaller scale, in order to improve the performance; applying grayscale to highlight contours; applying Gaussian filter to reduce the noise in order to clear the irrelevant shapes. The preprocessed image is then passed to the Suzuki Abe Border algorithm, from OpenCV library that will be responsible to identify the searched shapes and their position inside the image.

For obtaining better results in ROI identification, we combine another search, based on the color, with the first approach. After filtering and merging the results from the two operations (the shapes identified in both are kept one single time), we proceed to the extraction of ROI based on the shapes coordinates.

Targets Recognition service is responsible for the recognition of traffic signs, by going through the areas of interest, returned from the Segmentation Service. Targets Recognition service is based on the 3D Registration template matching approach, which compares the received input frame against a set of predefined images, called models. In [10] is specified that the template matching approach uses information extracted from real object images to identify the 
corresponding pattern. Moreover, the authors highlight that this method can recognize partial occurrences. 3D Registration method consists in adding virtual 3D elements into a real scene, based on the information received from the tracking process. This process computes in real time the position and the posture of the device within the real scene. Moreover, 3D registration method is based on marker-less approach in order to identify real object, like: buildings, posters, signs and other elements.

The main responsibilities of the AR service are:

- collects information about the position of the car, using the GPS;

- ensures integration between the mobile application and the back-end system, in order to obtain the virtual elements

- projects the augmented reality scene on the mobile device screen

- provides a visual interface to the user, ensuring a good communication between him and the system

Searching Service contains a number of methods that are based on REST principles to facilitate communication between $A R$ Service and AR Database. Those methods are responsible to extract virtual elements stored in the AR Database corresponding to the road signs identified by the Targets Recognition Service. This data represents the virtual information used by the AR Service to compose the virtual environment.

\section{Using ontologies for improved recommendations}

After detecting the traffic sign and augmenting the scene on the mobile device screen, we will also need to interpret the image and help the driver with an audio recommendation. Basically we need to attach some knowledge to each traffic sign, to guide its interpretation, and this can be achieved by the use of ontologies. Ontologies help to link data with a formal semantics, labelling it with information related to its meaning and context, which can be used to draw conclusions. Having this knowledge proves to be a necessity for a context-aware driver assistance system.

There are several researches that employ the use of ontologies for better results in traffic sign recognition and interpretation. In study [11], the authors propose an ontology to establish an open source semantic knowledge base with traffic signs and the traffic rules they dictate. Although the ontology is currently populated with road signs from Romania, they claim that foreign signs can also be added, relying on the $r d f$ :langString property in order to identify the country in which the sign or the traffic rule is used. For extending the ontology with signs that their system fails to identify, gamification technique is used, to motivate the user to categorize the sign and link it with the appropriate applicable traffic rules.

Moreover, the authors of [12] present an ontology-based context-model for representation of driving scenarios. In order to facilitate a common information representation and exchange process between collaborating vehicles, OWL was chosen for modelling the ontology. The context of a driving situation is expressed as a combination of other four major sub-contexts: the operating environment of the vehicle, the driver, the vehicle with the built-in driver assistance system and national traffic regulations. The classes in the ontology represent traffic objects from the previous four context categories, together with their attributes and relationships.

Furthermore, the authors of [13] propose an ontology-based framework for assessing the degree of risk in a road scene. Taking into account that the road users do not always obey the traffic rules, the novelty of the framework stems from its ability to interpret unpredictable road traffic. Input data is taken from a monocular video camera only that is capturing scene views from the driver's perspective. The proposed ontology consists of three main classes which correspond to factors contributing to risk: risk from object, environmental risk and road environmental 
risk. Each of these classes are in relation with other sub-classes. Road traffic signs are part of the road environmental risk. Combining information from the risk factor classes and using SWRL (semantic web rule language), inference rules for risk assessment are created.

The authors of [14] propose and test a traffic sign recognition system in poor conditions applied on the road signs in Thailand. After performance analysis they concluded that next step in future work is to apply ontology-based knowledge in order to improve traffic sign recognition. In favour of guiding the interpretation of the traffic signs we propose the ontology depicted in Figure 2. The main intention of the developed ontology is to provide relevant safety information to drivers about the traffic signs encountered on the road.

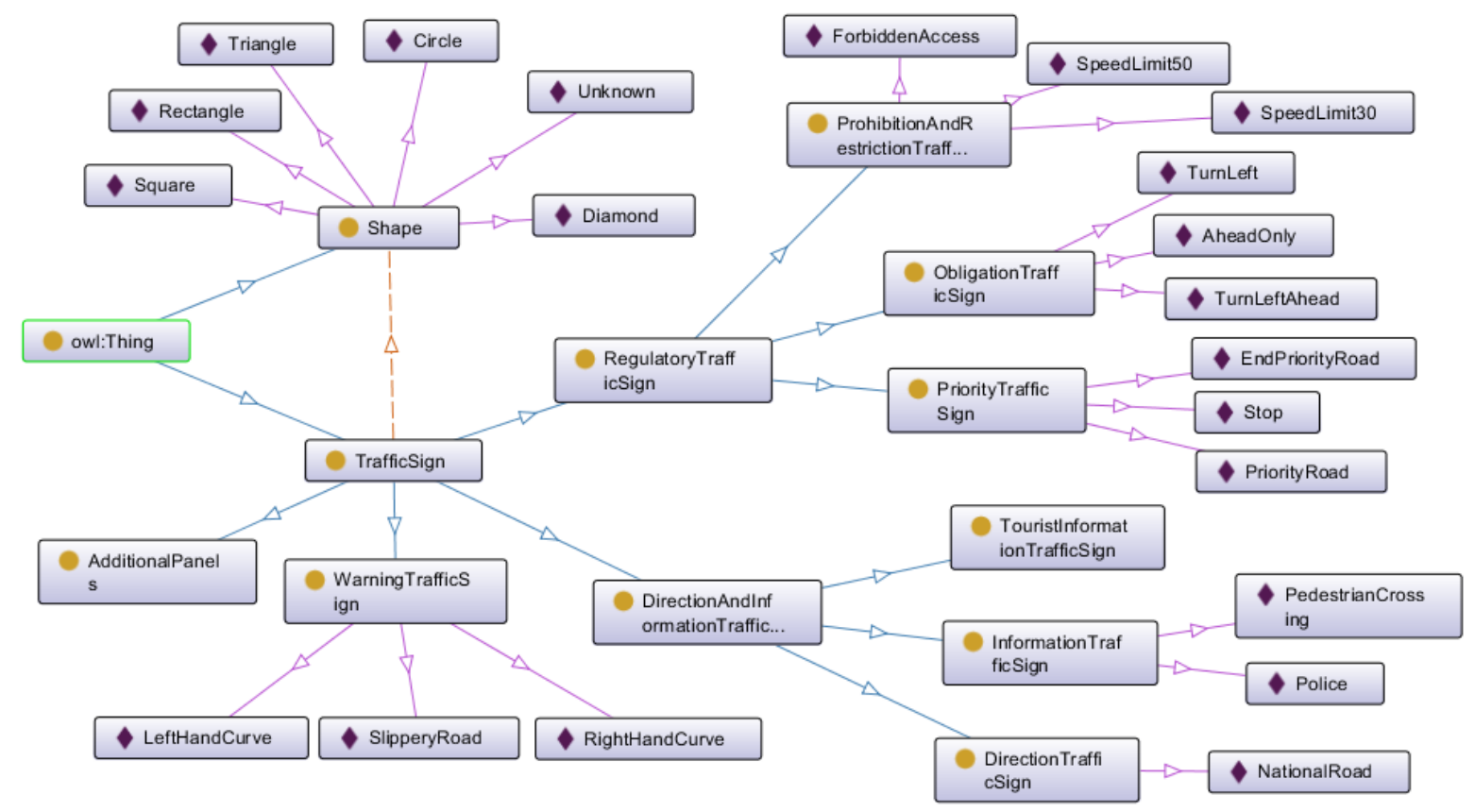

Figure 10. The proposed traffic signs ontology (displayed in OntoGraph, Protégé)

The main entity of the ontology is TrafficSign. Each traffic sign has a shape, represented through the Shape entity that has several individuals, like: Circle, Triangle, Square, Rectangle, Diamond and Unknown. Also we added a description datatype property for the TrafficSign class. This is a string literal and it will be used to understand the meaning of the road sign. It will also be added for different languages and will be used for text to speech recommendations. We split the road signs in four major classes, each having their own sub-classes and individuals, as per classification found on [15]. RegulatoryTrafficSign class is composed of ProhibitionAndRestrictionTrafficSign (with individuals like ForbiddenAccess, SpeedLimit30, SpeedLimit50), ObligationTrafficSign (with individuals like TurnLeft, TurnLeftAhead, AheadOnly) and PriorityTrafficSign (with individuals like PriorityRoad, EndPriorityRoad, Stop). DirrectionAndInformationTrafficSign class has as sub-classes InformationTrafficSign (with individuals like PedestrianCrossing, Police), DirectionTrafficSign (with individuals like NationalRoad) and TouristInformationTrafficSign. Last two road sign classes are WarningTrafficSign (with individuals like LeftHandCurve, RightHandCurve, SplipperyRoad) and AdditionalPanels. All the entities and their properties, presented as a part of the designed ontology, are used to represent the data about the road signs, in order to enable searching based on the semantics of their underlying content. For querying the ontology and extract information about a particular traffic sign, SPARQL (SPARQL Protocol and RDF Query Language) is employed. 
www.conferenceie.ase.ro

\section{Influence factors}

The purpose of the current section is to present the main issues identified during the development and testing of the proposed solution. Moreover, Figure 1 illustrates the result of using the application, described in Section 2, in real world. We focused primarily on speed limit traffic signs. The conducted test aimed to identify a single traffic sign, shown in Figure 3 , in good lighting conditions with a mobile device which has the following features: Samsung Galaxy S9, Exynos 9810 Octa Core, Android, F1.5/F2.4, 12 Mb camera resolution.

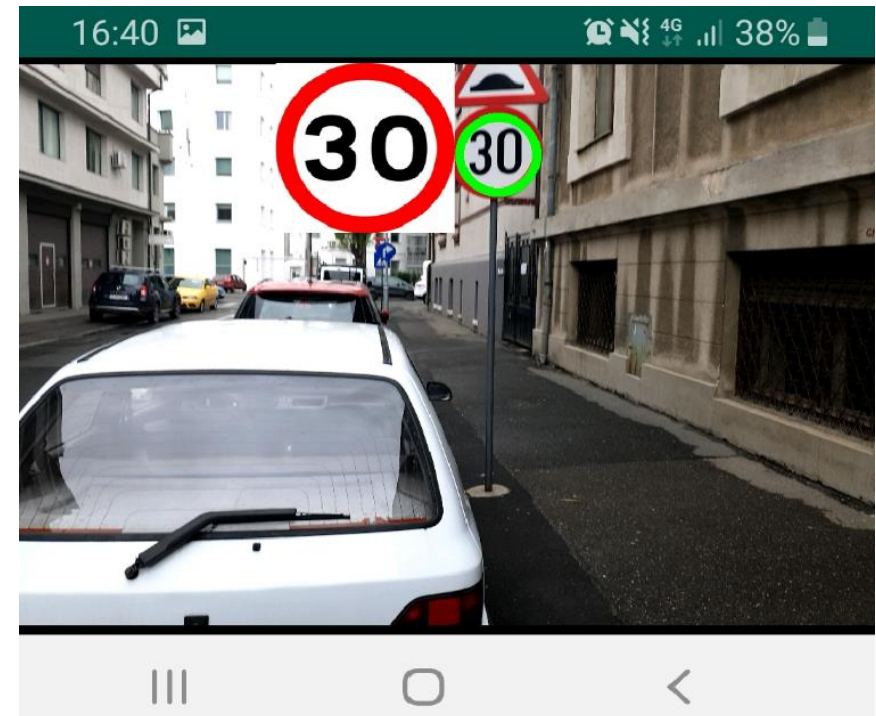

Figure 11. Usage of the proposed prototype

Nevertheless, the performance of template-matching approach of 3D Registration technique, which underpins the traffic sign recognition service, is affected by several factors, such as: video source, lighting conditions, fading and blurring effect, affected visibility, scene complexity, occlusion, chaotic background and viewing angle, deterioration and deformations of the traffic signs, speed of vehicles and variable size of the traffic signs.

Video source - the process of recognition depends on the quality of the video camera attached to the mobile device. Problems may also occur if the mobile device is not attached to the car's board, which can lead to distortion of video sequences due to turbulence. Another problem is caused by the autofocus mode of the camcorder.

Lighting conditions - image quality is influenced both by the light source (sunlight, artificial light from the bulbs) and by its intensity (day or night), which makes this factor one of the biggest problems of the service recognition from the proposed prototype.

Fading and blurring effect - poor meteorological conditions combined with sunlight lead to erroneous results of the identification service regarding the areas of interest. In [16] it is suggested to use Hough transformation which is robust in different lighting conditions and illumination.

Scenarios complexity - multiple occurrences of signs that have the same shape or custom objects with similar structure result in erroneous results of the target recognition service. Also, the detection process is also affected by partial signs overlapping, rotation or scaling.

Occlusion - any object that affects the visibility of traffic signs, such as trees, parked vehicles, etc. leads to lower performance of the process that identifies the areas of interest.

Deterioration and deformation of traffic signs - traffic signs may be partially or totally damaged. This affects both the region of interest identification service and the recognition 
service, considering the fact that the last one is based on the template-matching approach, which focuses mainly on comparing the form.

Chaotic background and viewing angle - The position of the car or mobile device in traffic can lead to obtaining video sequences in different angles of the road signs that affect the detection process. Also, the existence in the background of traffic signs of other objects, with similar shapes or colors, causes an overlap of colors, which makes the proposed system unable to precisely identify the areas of interest.

Speed of vehicles affects the quality of video sequences which in turn affects the process of recognizing road signs.

Since all of the above factors affect to a certain degree the performance of the augmented reality prototype for traffic sign recognition, it was necessary to use the following methods trying to reduce their effects: image resizing, grayscale transformation, median blur, Hough transformation.

\section{Conclusions and future work}

Considering that more than $80 \%$ of vehicle accidents are due to human error, this paper proposes applying augmented reality techniques in order to increase driver awareness in traffic. We introduced a traffic signs recognition prototype based on template matching approach of the 3D Registration technique in order to make use of the benefits provided by mobile augmented reality. The purpose of the solution is to assist the drivers during the driving process by augmenting additional audio and visual information with the aim to reduce the number of accidents. For understanding the meaning of each traffic sign we designed an ontology, queried through SPARQL.

While testing the prototype in a real world environment a series of factors that affect the performance of the application were identified: video source, lighting conditions, fading and blurring effect, affected visibility, scene complexity, occlusion, chaotic background and viewing angle, deterioration and deformations of the traffic signs, speed of vehicles and variable size of the traffic signs. While for the video quality solutions can be easily found replacing the mobile device with one that has better hardware specifications, lighting conditions are the ones that are the hardest to manage. In order to minimize the negative effects of the above mentioned factors we applied the following methods: image resizing, grayscale transformation, median blur, Hough transformation.

In the future work we aim to continue developing and improving the proposed architecture of the traffic signs recognition system based on mobile augmented reality. Beside speed limit traffic signs, we plan to include other road signs like stop and give away, as most of the accidents are caused by the drivers either not giving priority or not complying with regulatory speed. Also, we will continue to present the results as our research progresses. Moreover, we will take into consideration the possibility to implement a different solution based on text recognition in order to extend the usage of the current solution with informative road signs.

\section{References}

[1] L. Abdi, F. Abdallah and A. Meddeb, "In-Vehicle Augmented Reality Traffic Information System: A New Type of Communication between Driver and Vehicle", Procedia Computer Science, vol. 73, pp. 242-249, 2015.

[2] J. Mota, I. Ruiz-Rube, J. Dodero and I. Arnedillo-Sánchez, "Augmented reality mobile app development for all", Computers \& Electrical Engineering, vol. 65, pp. 250-260, 2018.

[3] R. Eyraud, E. Zibetti and T. Baccino, "Allocation of visual attention while driving with simulated augmented reality", Transportation Research Part F: Traffic Psychology and Behaviour, vol. 32, pp. 46-55, 2015. 
[4] F. Dita, "An analysis of different 3D Registration methods in augmented reality", in 16th International Conference on Informatics in Economy (IE 2017): Education, Research and Business Technologies, Bucharest, 2017, pp. 108-115.

[5] Y. Chen, Y. Xie and Y. Wang, "Detection and Recognition of Traffic Signs Based on HSV Vision Model and Shape features", Journal of Computers, vol. 8, no. 5, 2013.

[6] K. Lim, Y. Hong, Y. Choi and H. Byun, "Real-time traffic sign recognition based on a general purpose GPU and deep-learning", PLOS ONE, vol. 12, no. 3, p. e0173317, 2017.

[7] L. Abdi and A. Meddeb, "Deep learning traffic sign detection, recognition and augmentation", Proceedings of the Symposium on Applied Computing - SAC '17, 2017.

[8] E. Ginters, "Augmented reality use for cycling quality improvement", Procedia Computer Science, vol. 149, pp. 167-176, 2019.

[9] Transport Department Government of Jharkhland. Causes of Road Accidents. Internet: http://jhtransport.gov.in/causes-of-road-accidents.html [Apr. 10, 2019]

[10] R. Vock, A. Dieckmann, S. Ochmann and R. Klein, "Fast template matching and pose estimation in 3D point clouds", Computers \& Graphics, vol. 79, pp. 36-45, 2019.

[11] B. Iancu and A. Zamfiroiu, "Automating the Process of Traffic Orientation Through Mobile Devices And Ontologies", JADLeT Journal of Advanced DistributedLearning Technology, vol. 3, no. 7, pp. 64-74, 2015.

[12] S. Fuchs, S. Rass, B. Lamprecht, and K. Kyamakya, "A Model for Ontology-Based Scene Description for Context-Aware Driver Assistance Systems," Proceedings of the First International Conference on Ambient Media and Systems, 2008.

[13] M. Mohammad, I. Kaloskampis, Y. Hicks and R. Setchi, "Ontology-based Framework for Risk Assessment in Road Scenes Using Videos", Procedia Computer Science, vol. 60, pp. 1532-1541, 2015.

[14] T. Surinwarangkoon, S. Nitsuwat and E. Moore, "Traffic Sign Recognition System for Roadside Images in Poor Condition", International Journal of Machine Learning and Computing, pp. 121-126, 2013.

[15] "Semne de circulatie explicate - DRPCIV", I-drpciv.ro, 2019. Internet: https://www.idrpciv.ro/semne-de-circulatie [Apr. 10, 2019]

[16] S. Wali, "Comparative Survey on Traffic Sign Detection and Recognition: a Review", PRZEGLĄD ELEKTROTECHNICZNY, vol. 1, no. 12, pp. 40-44, 2015. 